BULLETIN OF THE

AMERICAN MATHEMATICAL SOCIETY

Volume 80, Number 3, May 1974

\title{
VECTOR FIELDS GENERATE FEW DIFFEOMORPHISMS
}

\author{
BY J. PALIS ${ }^{1}$ \\ Communicated by C. Moore, June 25, 1973
}

Let $M$ be a compact $C^{\infty}$ manifold without boundary. Let $\operatorname{Diff}^{r}(M)$ be the group of $C^{r}$ diffeomorphisms of $M$ with the $C^{r}$ topology. If $r=0$, this corresponds to the group of homeomorphisms of $M$. A $C^{s}$ flow on $M$ is a continuous group homomorphism $\varphi: R \rightarrow \operatorname{Diff}^{s}(M), 0 \leqq s \leqq \infty$. In a natural way, $C^{1}$ vector fields generate $C^{1}$ flows and Lipschitz vector fields generate $C^{0}$ (topological) flows. We say that $f \in \operatorname{Diff}^{r}(M)$ embeds in a $C^{s}$ flow, $s \leqq r$, if $f$ is the map at time one of such a flow.

Our main purpose is to announce results showing that few diffeomorphisms, in the sense of Baire category, embed in flows or are generated by vector fields with some mild differentiability or Lipschitz condition. Here we will prove only one of these results concerning flows generated by vector fields.

Several authors have treated similar questions. For $C^{2}$ diffeomorphisms of the circle, our last theorem follows from stronger results of Kopell [2] and it was also proved in [4], where more references can be found. The embedding of diffeomorphisms in topological flows was also considered in [5].

The author acknowledges very useful conversations with C. Pugh and M. Shub and several people at IMPA.

We now show that with a mild assumption on the vector fields, the diffeomorphisms they possibly generate form a subset of first category in $\operatorname{Diff}^{1}(M)$.

Fix a riemannian metric on $M$. Let $x$ be a singularity for a vector field $X$. $X$ is said to be Lipschitz at $x$ if there exists a constant $K>0$ such that $|X(y)| \leqq K d(x, y)$ for every $y \in M$, where $d(x, y)$ is the distance between $x$ and $y$. Let $\chi$ denote the set of $C^{0}$ vector fields on $M$ that generate topological flows and are Lipschitz at the singularities.

TheOREM. The subset $F=\left\{f \in \operatorname{Diff}^{1}(M) \mid f=X_{t=1}\right.$ for some $\left.X \in \chi\right\}$ is of first category in $\operatorname{Diff}^{1}(M)$.

Proof. Consider the set $B$ of Kupka-Smale diffeomorphisms whose periodic points are dense in the nonwandering set. Pugh [6] showed that $B$ is of second category in $\operatorname{Diff}^{1}(M)$. Following [5], if $f \in B$ and embeds in a topological flow then the periodic points of $f$ are in fact

AMS (MOS) subject classifications (1970). Primary 58F10.

${ }^{1}$ Guggenheim fellow. 
fixed. This reduces the theorem to a similar one in the set $S$ of MorseSmale diffeomorphisms whose nonwandering sets consist of fixed points. Notice that $S$ is open in $\operatorname{Diff}^{1}(M)$ [5].

Thus it is enough to show that $G=F \cap S$ is of first category in $S$. For each $n \in Z_{+}$consider a subset $G_{n} \subset S$ as follows. For $f \in G_{n}$ there exists a (not necessarily continuous) vector field $X$, invariant by $D f$ and with Lipschitz constant bounded by $n$ at the fixed points of $f$. Moreover, we require that $\|X\| \leqq n$, and $\|X\| \geqq 1 / n$ outside open discs of radius $r$ centered at these points. We take $r$ satisfying the relation $r(1+\|D f\|) \leqq d$, where $d$ is the minimum distance between the fixed points of $f$. This ensures that $X$ does not vanish on the orbits of $f$ except the fixed ones. We have that $G \subset \bigcup G_{n}$. Also each $G_{n}$ is closed. We now show that $H=S-G_{n}$ is dense in $S$. Take any $\tilde{f} \in S$. We first approximate $\tilde{f}$ by $f \in S$ so that $f$ is linear near a sink $p$ and the proper values of $D f(p)$ satisfy the condition $\left|u_{1}\right| \leqq\left|u_{2}\right|<\left|u_{i}\right|$ for $i>2$ and $\left|u_{1}\right|=\left|u_{2}\right|$ only if $u_{1}$ is not real. Let $E$ be the local strong stable manifold of $p$. $E$ is a linear subspace and $\operatorname{dim} E=1$ if $u_{1}$ is real and $\operatorname{dim} E=2$ if not. We perform a local perturbation of $f$ parallel to $E$ to achieve an element $h \in H$. Assume $\operatorname{dim} E=2$. Then $f / E$ can be written in polar coordinates as $f(\rho, \varphi)=(a \rho, b+\varphi)$, with $p$ as the origin, $a>0$ and $0<b<2 \pi$. Set for the local expression of $h / E$

$$
\begin{aligned}
& h(0,0)=(0,0), \quad D h(0,0)=D f(0,0)=f, \\
& h(\rho, \varphi)=\left(a \rho+\frac{\rho}{\log \rho} \sin \left(2 \pi \frac{\log \rho}{\log a}\right), b+\varphi\right) .
\end{aligned}
$$

Such a map $h$ is $C^{1}$ and can be extended to be in $S, C^{1}$ near $f$ and in fact equal to $f$ off some neighborhood of $p$. The case $\operatorname{dim} E=1$ is similar and we repeat this construction near all sinks. We claim that $h \in S-G_{n}$. For suppose there is a vector field $X$ invariant by $D h$ and Lipschitz at the fixed points of $h$. Then $X$ must be tangent to $E$ near $p$. Take a point $y=\left(a^{n_{0}}, 0\right)$ in $E, n_{0} \in Z_{+}$large enough. For $X$ along the orbit of $y$, considering that the positive $h$-orbit and $f$-orbit of $y$ are the same, we have

$$
\frac{\left|X\left(h^{n}(y)\right)\right|}{\left|h^{n}(y)\right|}=\prod_{k=0}^{n-1}\left(1+\frac{2 \pi}{a \log ^{2} a} \cdot \frac{1}{n_{0}+k}\right) \frac{|X(y)|}{a^{n_{0}}}
$$

which diverges as $n \rightarrow \infty$ unless $X(y)=0$. Thus $h \in S-G_{n}$ and so $S-G_{n}$ is dense in $S$. This implies that $G \subset \bigcup G_{n}$ is of first category in $S$, proving the theorem.

The other results. The main one is

THEOREM. The subset of $\operatorname{Diff}^{1}(M)$ of diffeomorphisms that embed in $C^{1}$ flows is of first category. 
With different arguments we get the following theorem for $\operatorname{Diff}^{r}(M)$, $r \geqq 2$. Let $A \subset \operatorname{Diff}^{r}(M)$ be the open set of diffeomorphisms satisfying axiom $A$ and the no cycle property [8].

THEOREM. The subset of $A$ of $C^{r}$ diffeomorphisms that embed in $C^{1}$ flows is of first category.

As a corollary, we obtain a similar result for diffeomorphisms generated by $C^{\mathbf{1}}$ vector fields.

The proofs will appear elsewhere. We just remark that for this last theorem we make use of a linearization theorem in [1], which generalizes [9].

We also pose the following question, in the way of a conjecture in [3], [7]: Is the set of diffeomorphisms with discrete centralizer of second category in $\operatorname{Diff}^{r}(M)$ ? This question is interesting on its own and, again, it is likely that the case $r=1$ should be treated differently from the others.

\section{REFERENCES}

1. P. Hartman, On local homeomorphisms of Euclidean spaces, Bol. Soc. Mat. Mexicana (2) 5 (1960), 220-241. MR 25 \#5253.

2. N. Kopell, Commuting diffeomorphisms, Proc. Sympos. Pure Math., vol. 14, Amer. Math. Soc., Providence, R.I., 1970, pp. 165-184. MR 42 \#5285.

3. - Commuting diffeomorphisms, Sympos. Differential Equations and Dynamical Systems, Springer-Verlag, Berlin and New York, 1971, pp. 162-163.

4. P. F. Lam, Embedding a homeomorphism in a flow subject to differentiability conditions, Topological Dynamics, Benjamin, New York, 1968, pp. 319-333.

5. J. Palis, On Morse-Smale dynamical systems, Topology 8 (1969), 385-404. MR 39 \#7620.

6. C. Pugh, An improved closing lemma and a general density theorem, Amer. J. Math. 89 (1967), 1010-1021. MR 37 \#2257.

7. S. Smale, Differentiable dynamical systems, Bull. Amer. Math. Soc. 73 (1967), 747-817. MR 37 \#3598.

8. — The $\Omega$-stability theorem, Proc. Sympos. Pure Math., vol. 14, Amer. Math. Soc., Providence, R.I., 1970, pp. 289-297. MR 42 \#6852.

9. S. Sternberg, Local $C^{n}$ transformations of the real line, Duke Math. J. 24 (1957), 97-102. MR 21 \#1371.

Instituto de Matematica Pura e Aplicada (IMPA), Rio de Janeiro, Brasil

Department of Mathematics, University of California, Berkeley, California 94720 